\title{
Disordered Presentation of Paraneoplastic Pancreatitis, Polyarthritis, and Panniculitis Syndrome in a Patient with End-Stage Pancreatic Cancer
}

Melody Maarouf MHS ${ }^{a}$, Marilyn R. Wickenheiser MD ${ }^{b}$, James E. Sligh Jr. MD PhD ${ }^{b, c}$, Keliegh Culpepper MD ${ }^{\mathrm{d}}$, Vivian Y. Shi MD

aUniversity of Arizona, College of Medicine, AZ

bUniversity of Arizona, Department of Medicine, Division of Dermatology, AZ

'Southern Arizona VA Healthcare System, AZ

dDermpath Diagnostics, Tucson, AZ

\section{ABSTRACT}

Pancreatitis, Polyarthritis, and Panniculitis (PPP) syndrome is a rare triad with variable initial presentations. We report a case of PPP due to stage IV pancreatic cancer presenting with polyarthritis following chemotherapy induction, whose diagnosis was delayed due to late manifestation of pancreatic panniculitis.

\section{CASE REPORT}

A 53-year-old male presented with a onemonth history of worsening polyarthralgia involving all joints of the upper and lower extremities. He was diagnosed with stage IV unresectable beta-islet neuroendocrine pancreatic cancer with liver metastasis 5 months prior, and had been receiving chemotherapy (capecitabine/temozolamide) for 1 month.

Clinical examination revealed diffusely tender joints involving hands, feet, knees, and elbows. Vital signs, cardiopulmonary and abdominal exams were unremarkable. Initial laboratory investigation revealed leukocytosis (WBC 34.4 1000/uL, Neutrophils $91 \%)$, elevated lipase $(25,560$ $\mathrm{U} / \mathrm{L}$ ) and inflammatory indices (CRP: 27.05 mg/dL; ESR 48 MM/HR; LDH 503 IU/L).
Autoimmunity (ANA/Rheumatoid Factor) and infectious disease workup (HIV, HCV, HBV, Gonorrhea/Chlamydia, Cryptococcus, Brucella, Lyme, and Coccidioidomycosis) were negative. Serum Rickettsial IgG was weakly positive, and lgM was negative. Serum uric acid, urine uric acid, and triglycerides were normal. MRI revealed cellulitis with a soft tissue ulcer on the left ankle and abscesses suspicious for septic tenosynovitis on the right wrist. Bacterial and fungal cultures of knee, ankle, and elbow joint aspirations were negative except for the single most painful joint, which expressed a purulent aspirate growing few gram-positive cocci. Initial treatment for suspected septic arthritis with broad-spectrum antibiotics, prednisone, NSAIDS, and opiates inadequately controlled his symptoms. Excruciating pain prevented activities of daily living, prompting hospital admission. 


\section{SKIN}

Approximately one month after onset of polyarthralgia, tender erythematous plaques (Figure 1) developed on the posterior legs. Histopathologic examination of punch biopsy confirmed lobular panniculitis with necrosis of adipocytes and a mixed inflammatory infiltrate (Figure 2).

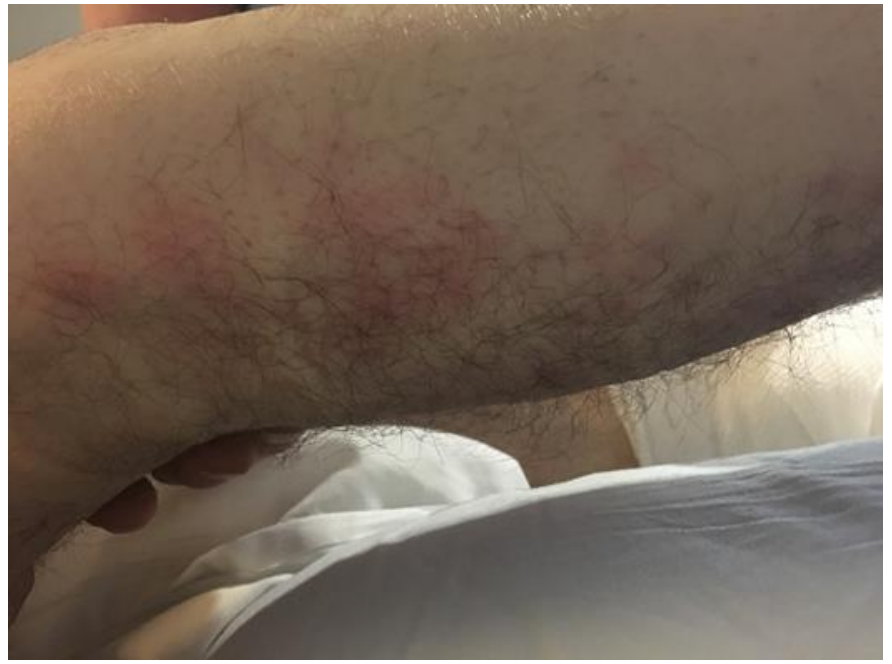

Figure 1. Clinical manifestation of pancreatic panniculitis: The clinical presentation of pancreatic panniculitis is characterized by erythematous, ill-defined, reddish-brown macules or nodules that may be painful or painless.

A

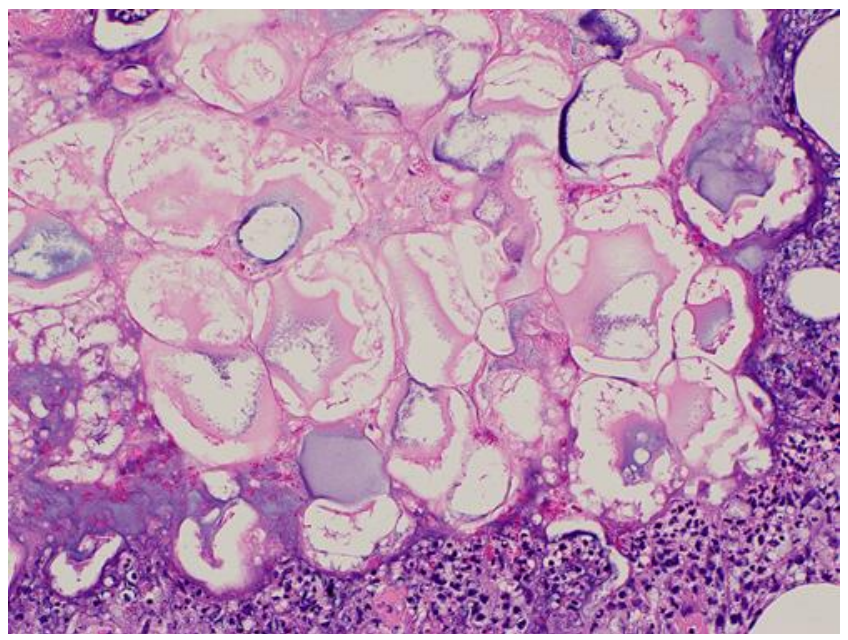

B

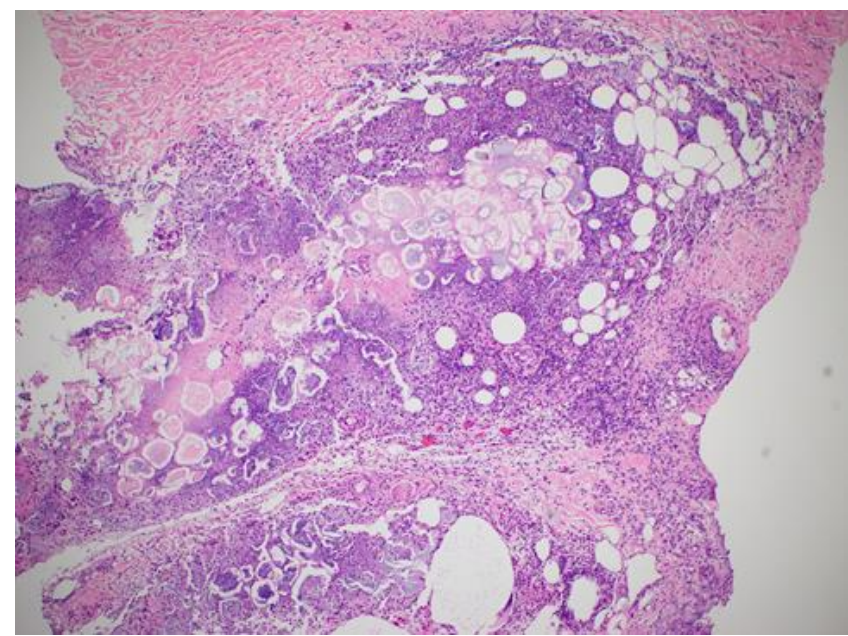

Figure 2. Histopathologic images of pancreatic panniculitis. A. The pattern of adipocyte necrosis, known as "ghost cells," is characteristic of pancreatic panniculitis. B. Perifollicular adipose tissue is also involved. 


\section{DISCUSSION}

The triad of pancreatic disease, panniculitis, and polyarthritis is referred to as PPP syndrome, which occurs in $0.3-1 \%$ of patients with benign or malignant pancreatic disease. ${ }^{1}$ Acute or chronic pancreatitis is the most commonly implicated pancreatic disorder, however, of the malignancies, acinar cell carcinoma is the most common culprit. Rarely, islet cell carcinomas are associated, and represent only $1-2 \%$ of PPP cases. Relation to carcinoma renders PPP syndrome a paraneoplastic phenomenon, ${ }^{2}$ and is a hallmark of poor prognosis, with a 4.75-months median survival after initial cutaneous eruption. ${ }^{1}$

PPP syndrome is typically diagnosed clinically. ${ }^{5}$ Nevertheless, histopathologic findings of pancreatic panniculitis include adipocyte destruction and necrosis, which results in "ghost cells" that lack nuclei and may have basophilic granular cytoplasm from calcium deposition via saponification. Panniculitis characteristically appears as tender, erythematous to red-brown nodules, frequently present on the lower extremities. In severe cases, nodules may ulcerate and drain an oily, brown, sterile substance. Arthritis is reported in approximately $56 \%$ of patients with PPP syndrome, and most commonly affects small joints of the hands, wrists, and feet. Joint aspirate typically yields a purulent appearing fluid that lacks white cells or microorganisms, but may include lipid liquid crystals. ${ }^{5}$ In $45 \%$ of these patients, arthritis follows a chronic course with poor response to NSAIDS and immunosuppressants, and may persist even after normalization of serum pancreatic enzymes. ${ }^{7}$

The pathogenesis of PPP has not entirely been elucidated. The leading hypothesis suggests that pancreatic parenchyma disruption causes release of pancreatic enzymes into the circulation, hydrolyzing fat lobules into glycerol and free fatty acids.

These byproducts saponify adipose tissues, resulting in panniculitis, as well as polyarthritis via infiltration of the periarticular fat pads. ${ }^{3,4}$ This hypothesis is supported by concomitant panniculitis and polyarthritis in approximately $50 \%$ of patients who have a fistula within the pancreatic portal system. ${ }^{5}$ Notably, serum pancreatic enzyme levels do not always correlate with disease presence or severity. In in-vitro studies, incubation of human subcutaneous fat with serum containing high levels of pancreatic lipase, trypsin, and amylase has not yielded panniculitic pathology. ${ }^{6}$

Management of PPP syndrome is frequently aimed at treating the underlying pancreatic disease. ${ }^{8}$ Panniculitis typically resolves following the resolution of the acute inflammation of the pancreatic parenchyma. Thus, supportive treatment, such as fluids and pain control, is all that is required. Panniculitis associated with pancreatic carcinoma tends to persist due to the difficulty in treating pancreatic carcinoma. ${ }^{5}$ Patients with pancreatic carcinoma may require alternative therapy for symptomatic management. In a case report, Ocreotide has demonstrated potential in slowing progression and reversing manifestations of PPP associated with pancreatic carcinoma. However, the positive implications of this therapy have been difficult to replicate. ${ }^{9}$

Cutaneous manifestation is commonly the initial presenting symptom of PPP syndrome, and may precede detection of pancreatic pathology by months. ${ }^{5}$ The literature consistently reports patients presenting with polyarthralgia and concomitant cutaneous findings, but mild or absent abdominal symptoms. Thus 
pancreatic pathology is typically the last of the triad to be detected. ${ }^{8}$ The delay in diagnosing the etiology of the debilitating arthralgias in our patient is due to PPP syndromes' rarity and his disordered presentation. To our knowledge, our patient is the first case in which cutaneous manifestations appeared months later than the other components of the syndrome. Our patient presented with abdominal pain, with a work up that revealed pancreatic carcinoma. Joint pain occurred concurrently with the induction of chemotherapy. Cutaneous eruption of panniculitis occurred two months following joint pain presentation. It is notable that the patient developed arthralgias and cutaneous manifestations following the initiation of chemotherapy. The overwhelming lysis of tumor cells from chemotherapy could have led to destruction of pancreatic parenchyma, and increased distribution of pancreatic enzymes.

This case raises awareness for possible pancreatic disease or malignancy in the presence of polyarthralgia and panniculitis. Our case highlights the potential for delayed cutaneous manifestation in PPP. Prompt diagnosis can allow judicious treatment of pancreatic pathology and prompt implementation of appropriate therapy.

Conflict of Interest Disclosures: no relevant conflict of interest.

Funding: none.

Corresponding Author:

Vivian Y. Shi, MD

Assistant Professor of Medicine (Dermatology

Division)

Arizona Cancer Center

1515 N. Campbell Ave. (PO Box 245024)

Building \#222, Levy Bldg., 1906E

Tucson, AZ 85724-5024

520-626-6024 (Office)

520-626-6033 (Fax)

vshi@email.arizona.edu

\section{References:}

1. Zhang G, Cao Z, Yang G, Wu W, Zhang T, Zhao Y. Pancreatic panniculitis associated with pancreatic carcinoma: A case report. Medicine (Baltimore). 2016;95(31):e4374.

2. Borowicz J, Morrison M, Hogan D, Miller R. Subcutaneous fat necrosis/panniculitis and polyarthritis associated with acinar cell carcinoma of the pancreas: a rare presentation of pancreatitis, panniculitis and polyarthritis syndrome. J Drugs Dermatol. 2010;9(9):1145-1150.

3. Zundler S, Erber R, Agaimy A, et al. Pancreatic panniculitis in a patient with pancreatic-type acinar cell carcinoma of the liver--case report and review of literature. BMC Cancer. 2016;16:130.

4. Fraisse T, Boutet O, Tron AM, Prieur E. Pancreatitis, panniculitis, polyarthritis syndrome: an unusual cause of destructive polyarthritis. Joint Bone Spine. 2010;77(6):617-618.

5. Garcia-Romero D, Vanaclocha F. Pancreatic panniculitis. Dermatol Clin. 2008;26(4):465-470, vi.

6. Requena L, Sanchez Yus E. Panniculitis. Part II. Mostly lobular panniculitis. J Am Acad Dermatol. 2001;45(3):325-361; quiz 362-324.

7. Narvaez J, Bianchi MM, Santo P, et al. Pancreatitis, panniculitis, and polyarthritis. Semin Arthritis Rheum. 2010;39(5):417-423. 
8. Ferri V, Ielpo B, Duran H, et al.

Pancreatic disease, panniculitis,

polyarthrtitis syndrome successfully treated with total pancreatectomy: Case report and literature review. Int J Surg Case Rep. 2016;28:223-226.

9. Price-Forbes AN, Filer A, Udeshi UL, Rai A. Progression of imaging in pancreatitis panniculitis polyarthritis (PPP) syndrome. Scand J Rheumatol. 2006;35(1):72-74. 